\title{
A Note on Congruent Numbers
}

\author{
By H. J. Godwin
}

Abstract. The list of known congruent numbers less than 1000 is extended by a proof that 19 primes of the form $8 k+7$ are congruent.

The positive rational integer $a$ is called congruent if and only if there exist rational nonzero integers $x, y, z, t$ such that $x^{2}-a y^{2}=z^{2}, x^{2}+a y^{2}=t^{2}$. (This is the definition in [3] where the history of early work on such numbers is given; Sierpiński [4] requires, unusually, that $y$ should be 1.) The most recent work on congruent numbers appears to be that of Alter and Curtz [1], who list the numbers $<1000$ known to be congruent or incongruent. In [1] 457 appears in both lists but, as noted later by the same authors [2], is in fact congruent.

In the present note a simple method, which enables a number of additions to be made to the list of congruent numbers, is described.

In what follows $p$ denotes a prime congruent to $7(\bmod 8)$.

If $p$ is congruent, we have $2 x^{2}=z^{2}+t^{2}=2\left((1 / 2 z+1 / 2 t)^{2}+(-1 / 2 z+1 / 2 t)^{2}\right)$, $2 p y^{2}=t^{2}-z^{2}$.

Hence, $x=l^{2}+m^{2}, z+t=2\left(l^{2}-m^{2}\right), t-z=4 l m$, or $x=l^{2}+m^{2}, z+t$ $=4 l m, t-z=2\left(l^{2}-m^{2}\right)$; whence $p y^{2}=\operatorname{lm}\left(l^{2}-m^{2}\right)$.

Hence, just one of $l, m, l-m, l+m$ is $p$ times a square, and the rest are squares. Also, since we may suppose that $(x, y)=1$, we have $(t, z)=1$ and so no two of the squares have a common factor.

Consideration of congruences modulo 8 gives either

I. $l=a^{2}, m=p b^{2}, l-m=c^{2}, l+m=d^{2}$, or

II. $l=a^{2}, m=b^{2}, l-m=p c^{2}, l+m=d^{2}$.

In case I we have $a^{2}-p b^{2}=c^{2}, a^{2}+p b^{2}=d^{2}$ and $a \leqslant a^{2}=l \leqslant l^{2}<x$ so that, by the method of descent, case I does not arise.

In case II we have, again by congruences modulo 8 , that $a$ is even and $b$ odd; and since $a^{2}+b^{2}=d^{2}$, we have

$$
a=2 e f, \quad b=e^{2}-f^{2}, \quad \text { whence } p c^{2}=\left(2 e f-e^{2}+f^{2}\right)\left(2 e f+e^{2}-f^{2}\right) .
$$

Since $(l, m)=1$, we have $(e, f)=1$ and so, since $e, f$ cannot both be odd because $b$ is odd, we have either

(i) $2 e f-e^{2}+f^{2}=p g^{2}, 2 e f+e^{2}-f^{2}=h^{2}$, or

(ii) $2 e f-e^{2}+f^{2}=g^{2}, 2 e f+e^{2}-f^{2}=p h^{2}$.

(i) gives $(e+f)^{2}=h^{2}+2 f^{2}$ and since $(e+f, f)=1$ we have

Received November 9, 1976.

AMS (MOS) subject classifications (1970). Primary 10B05; Secondary 65A05.

Key words and phrases. Congruent numbers, Diophantine equations.

Copyright $\odot$ 1978, American Mathematical Society 


$$
\begin{aligned}
e+f=r^{2}+2 s^{2}, \quad f=2 r s, \\
\quad \text { whence } p g^{2}=\left(r^{2}+2 s^{2}\right)^{2}-2\left(r^{2}-2 r s+2 s^{2}\right)^{2}=f(r, s) \text { say. }
\end{aligned}
$$

Case (ii) leads to the same result.

We now search for pairs $(r, s)$ such that the squarefree part of $f(r, s)$ is $p$.

Since $f(2 s, r)=4 f(r, s)$, it is sufficient to consider $r / s<\sqrt{ } 2$, and we have also

$$
r / s>t=\frac{\sqrt{ } 2-2 \sqrt{ }(\sqrt{ } 2-1)}{\sqrt{2}-1}=.3066 \ldots,
$$

the smaller zero of $f(x, 1)$. Since 2 is a quadratic residue of $p$, say $2 \equiv n^{2}(\bmod p)$, we have

$f(r, s) \equiv\left(\left(r^{2}+2 s^{2}\right)-n\left(r^{2}-2 r s+2 s^{2}\right)\right)\left(\left(r^{2}+2 s^{2}\right)+n\left(r^{2}-2 r s+2 s^{2}\right)\right)(\bmod p)$.

Since -1 is a quadratic nonresidue of $p$, just one of the quadratic factors is reducible modulo $p$, and so $r / s \equiv r_{1}$ or $r_{2}(\bmod p)$.

We now consider, for each $s$, the values $f(r, s)$ where $r=r_{1} s-k p$ or $r_{2} s-k p$ for

$$
t<r_{i}-k(p / s)<\sqrt{ } 2, \quad i=1,2 .
$$

A search, mainly by computer, gave the following values of $p, r, s$ and so each of the primes $p$ listed is congruent.

\begin{tabular}{lrr}
$p$ & $r$ & \multicolumn{1}{c}{$s$} \\
103 & 38 & 119 \\
127 & 136 & 443 \\
167 & 14 & 27 \\
191 & 60 & 193 \\
199 & 26 & 49 \\
223 & 256 & 301 \\
263 & 4017 & 12767 \\
271 & 49 & 106 \\
311 & 705 & 2061 \\
359 & 23 & 75 \\
383 & 153 & 138 \\
431 & 61 & 114 \\
439 & 130 & 127 \\
463 & 67 & 146 \\
487 & 751 & 1973 \\
631 & 2615 & 1927 \\
839 & 25 & 21 \\
919 & 142 & 143 \\
991 & 211 & 670
\end{tabular}

The primes $p<1000$ not in the above list are $367,503,599,607,647,727$, $743,823,863,887,911,967,983$, and for each of these a search for $s \leqslant 40,000$ 
yielded no value of $r$. However, the wide variation in the values of $s$ in the table does not preclude the existence of solutions with $s>40000$ and the conjecture (see $[1])$ that all numbers $\equiv 5,6$ or $7(\bmod 8)$ are congruent is not refuted.

Department of Statistics and Computer Science

Royal Holloway College

Egham Hill,

Egham, Surrey TW20 OEX, England

1. R. ALTER \& T. B. CURTZ, "A note on congruent numbers," Math. Comp., v. 28, 1974, pp. 303-305.

2. R. AlTER \& T. B. CURTZ, Corrigendum, Math. Comp., v. 30, 1976, p. 98.

3. L. E. DICKSON, History of the Theory of Numbers, Vol. 2, New York, 1952, pp. 459472. (Reprint.)

4. W. SIERPINSKI, Elementary Theory of Numbers, PWN, Warsaw, 1964. 